\title{
A Study on Propagation of Waves in a Transversely Isotropic Poroelastic Layer Bounded between Two Viscous Liquids
}

\author{
Nageswaranath Chinta1, Ahmed Shah Syed², Ramesh Modem³, \\ Venkata Ramanamurthy Mangipudi ${ }^{4}$
}

\author{
${ }^{1}$ Department of Mathematics, BVRITH College of Engineering for Women, Hyderabad, India \\ ${ }^{2}$ Department of Mathematics, Deccan College of Engineering and Technology, Hyderabad, India \\ ${ }^{3}$ Department of Mathematics, University College of Technology, Osmania University, Hyderabad, India \\ ${ }^{4}$ Department of Mathematics, Osmania University, Hyderabad, India \\ Email: nagesh.nath@rediffmail.com
}

How to cite this paper: Chinta, N., Syed, A.S., Modem, R. and Mangipudi, V.R. (2019) A Study on Propagation of Waves in a Transversely Isotropic Poroelastic Layer Bounded between Two Viscous Liquids. Open Journal of Acoustics, 9, 1-12. https://doi.org/10.4236/oja.2019.91001

Received: December 21, 2018

Accepted: March 3, 2019

Published: March 6, 2019

Copyright $\odot 2019$ by author(s) and Scientific Research Publishing Inc. This work is licensed under the Creative Commons Attribution International License (CC BY 4.0).

http://creativecommons.org/licenses/by/4.0/

\begin{abstract}
Propagation of Love waves in a transversely isotropic poroelastic layer bounded between two compressible viscous liquids is presented. The equations of motion in a transversely isotropic poroelastic solid are formulated in the framework of Biot's theory. A closed-form solution for the propagation of Love waves is obtained in a transversely isotropic poroelastic layer. The complex frequency equation for phase velocity and attenuation of Love waves is derived for a transversely isotropic poroelastic layer when it is bounded between two viscous liquids and the results are compared with that of the poroelastic layer. The effect of viscous liquids on the propagation of Love waves is discussed. It is observed that the presence of viscous liquids decreases phase velocity in both transversely isotropic poroelastic layer and poroelastic layer. Results related to the case without viscous liquids have been compared with some of the earlier results and comparison shows good agreement.
\end{abstract}

\section{Keywords}

Viscosity, Love Waves, Wave Number, Dissipation Coefficient, Transversely Isotropic Poroelastic Layer

\section{Introduction}

Deresiewicz [1] [2] discussed propagation of elastic waves in non-dissipative porous solid. A study on propagation of Love waves in a compressible viscous liquid layer bounded between an elastic layer and homogeneous isotropic elastic 
half-space was done by Bhangar [3]. Phase velocity, group velocity, quality factor $(Q)$ and displacement in the elastic layer and half space have been computed as a function of the frequency for various values of the ratio of thickness to coefficient of viscosity. It is shown that Love waves are not attenuated when the ratio takes the values zero and infinity. Also, the quality factor attains minimum value as a function of dimensionless angular frequency that the ratio is not equal to zero or infinity. Two dimensional wave propagation in a viscous liquid layer bounded between two poroelastic half-spaces was investigated by Nageswara Nath et al. [4]. Three types of bonding, i.e. welded interface, smooth interface and loosely bonded interface, are considered. The frequency equation of the interfacial waves for each of the above three types of bonding for infinite wavelength is obtained and it is observed that the frequency equation is independent of the nature of bonding. Love wave propagation in the elastic layered waveguide loaded with a viscous Newtonian liquid on the surface is investigated by Kiełczynski et.al. [5]. Love wave amplitude distribution as a function of depth has been determined. It was observed that for a viscous liquid loading, the amplitude of the wave changed with depth in an oscillatory way, and decayed to zero. The effect of gravity on propagation of Love waves in a fluid-saturated porous layer bounded above by a rigid boundary and below by an elastic half-space under gravity has been discussed by Anjana et al. [6]. It is observed that there is a significant effect of gravity, porosity and anisotropy simultaneously in the propagation of Love waves. The phase velocity of Love wave is less in the gravitating medium in comparison to that of non-gravitating medium. Wang and Zhang [7] studied Love wave propagation transversely isotropic poroelastic layered half-space. The frequency equation for the phase velocity and attenuation is solved using an iterative method. It is shown that the solution depends upon a parameter involving the critical frequency and the thickness of the layer. The attenuation vanishes at extreme values of frequency i.e. at zero frequency or infinite frequency. The effect of viscosity on propagation of Love waves in a fluid loaded transversely isotropic poroelastic layered half-space was studied by Nageswarnath et al. [8]. Phase velocity of Love waves is more for higher values of coefficients of viscosity in case of fluid loaded transversely isotropic poroelastic layered half-space. Attenuation is more for lower values of frequency and it is steady with an increase in viscosity. Also, the phase velocity is highest when two solids are transversely poroelastic and it is lowest when two solids are just poroelastic. Nageswaranath et al. [9] discussed propagation of Love waves in viscous liquid layer bounded between poroelastic layer and half-space which was transversely isotropic. It is observed that there is no influence of coefficient of viscosity of liquid layer on phase velocity when the liquid layer is bounded between poroelastic layer and half-space. Kundu et al. [10] investigated the effect of initial stress on Love waves propagating in a homogeneous layer over a porous half-space with irregular boundary. It is observed that propagation of Love wave is influenced by initial stress parameters, corrugation 
parameter and porosity of half-space. Particularly, it is found that the phase velocity of waves decreases with increase in non-dimensional wave number, initial stress parameters and porosity of half-space.

In the present analysis, propagation of Love waves in a transversely isotropic poroelastic layer bounded between two viscous liquids is discussed in the frame of work-Biot's theory. The governing equations of motion in a transversely isotropic poroelastic layer are derived. The frequency equation for Love waves in a transversely isotropic poroelastic layer bounded between two viscous liquids is obtained and the results are compared with that of poroelastic layer. Phase velocity has been computed and analyzed against non-dimensional wavenumber. The effect of presence of viscous liquids is studied and it is observed that presence of viscous liquids decreases phase velocity of Love waves in transversely isotropic poroelastic layer and in poroelastic layer. Also, it is observed that phase velocity for transversely isotropic poroelastic layer is more compared to poroelastic layer. In general, phase velocity decreases as wave number increases.

\section{Basic Equations, Formulation and Solution of the Problem}

Consider a rectangular co-ordinate system $(x, y, z)$ with $x$ and $y$ axes taken as horizontal and $z$-axis as positive downwards normal to the plane. Propagation of waves is taken as two-dimensional (i.e. propagation in $x z$-plane) along the $x$ direction. A transversely isotropic poroelastic layer of thickness ' $h$ ' bounded between two compressible viscous liquids is considered. The boundaries of the poroelastic layer are taken as $z=0$ and $z=h$. At $z=0$ the poroelastic layer is interacting with upper viscous liquid, whereas at $z=h$ it is interacting with lower viscous liquid. The physical parameters of two viscous liquids are denoted by a superscript $j(1,2)$ enclosed in parentheses. The parameters with superscript (1) \& (2) refer to upper viscous liquid and lower viscous liquid, respectively. The parameters of poroelastic layer are without any superscript. The geometry of the problem is shown in Figure 1.

The equations of motion of a homogeneous, transversely isotropic poroelastic solid in the presence of dissipation $b$ are

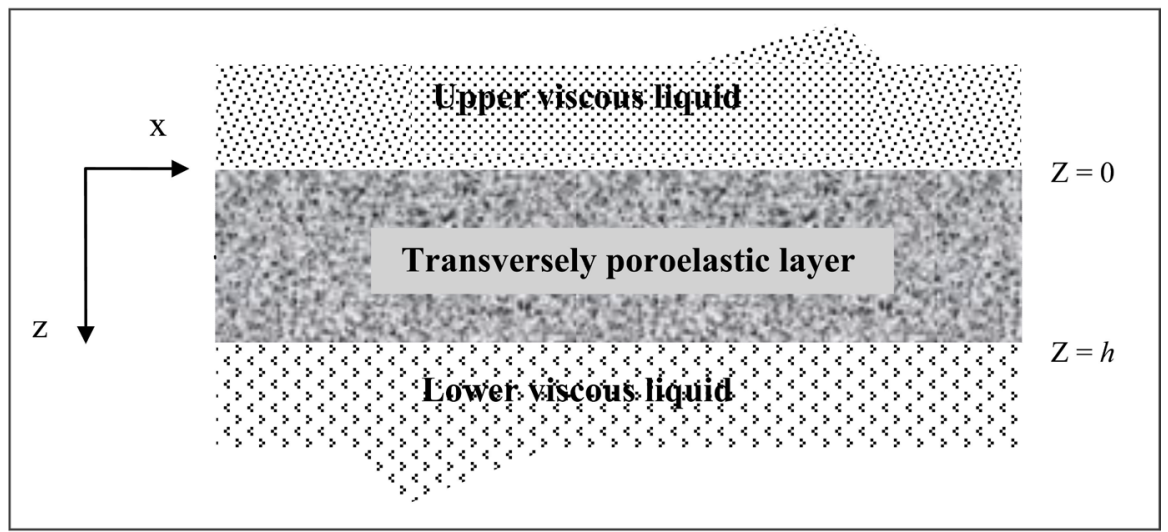

Figure 1. Geometry of the problem. 


$$
\begin{aligned}
& {\left[(A+2 N) \frac{\partial^{2}}{\partial x^{2}}+N \frac{\partial^{2}}{\partial y^{2}}+L \frac{\partial^{2}}{\partial z^{2}}\right] u_{x}+(A+2 N) \frac{\partial^{2} u_{y}}{\partial x \partial y}+(F+L) \frac{\partial^{2} u_{z}}{\partial x \partial y}+M \frac{\partial \in}{\partial x}} \\
& =\frac{\partial^{2}}{\partial t^{2}}\left(\rho_{11} u_{x}+\rho_{12} U_{x}\right)+b \frac{\partial}{\partial t}\left(u_{x}-U_{x}\right), \\
& M \frac{\partial^{2} u_{x}}{\partial x^{2}}+M \frac{\partial^{2} u_{y}}{\partial x \partial y}+Q \frac{\partial^{2} u_{z}}{\partial x \partial z}+R \frac{\partial \in}{\partial x}=\frac{\partial^{2}}{\partial t^{2}}\left(\rho_{12} u_{x}+\rho_{22} U_{x}\right)-b \frac{\partial}{\partial t}\left(u_{x}-U_{x}\right), \\
& (A+2 N) \frac{\partial^{2} u_{x}}{\partial x \partial y}+\left[N \frac{\partial^{2}}{\partial x^{2}}+(A+2 N) \frac{\partial^{2}}{\partial y^{2}}+L \frac{\partial^{2}}{\partial z^{2}}\right] u_{y}+(F+L) \frac{\partial^{2} u_{z}}{\partial y \partial z}+M \frac{\partial \in}{\partial y} \\
& =\frac{\partial^{2}}{\partial t^{2}}\left(\rho_{11} u_{y}+\rho_{12} U_{y}\right)+b \frac{\partial}{\partial t}\left(u_{y}-U_{y}\right), \\
& M \frac{\partial^{2} u_{x}}{\partial x \partial y}+M \frac{\partial^{2} u_{y}}{\partial y^{2}}+Q \frac{\partial^{2} u_{z}}{\partial y \partial z}+R \frac{\partial \in}{\partial y}=\frac{\partial^{2}}{\partial t^{2}}\left(\rho_{12} u_{y}+\rho_{22} U_{y}\right)-b \frac{\partial}{\partial t}\left(u_{y}-U_{y}\right), \\
& (L+F) \frac{\partial^{2} u_{x}}{\partial x \partial y}+(L+F) \frac{\partial^{2} u_{y}}{\partial y \partial z}+\left[L \frac{\partial^{2}}{\partial x^{2}}+L \frac{\partial^{2}}{\partial y^{2}}+C \frac{\partial^{2}}{\partial z^{2}}\right] u_{z}+Q \frac{\partial \in}{\partial z} \\
& =\frac{\partial^{2}}{\partial t^{2}}\left(\rho_{11} u_{z}+\rho_{12} U_{z}\right)+b \frac{\partial}{\partial t}\left(u_{z}-U_{z}\right), \\
& M \frac{\partial^{2} u_{x}}{\partial x \partial z}+M \frac{\partial^{2} u_{y}}{\partial y \partial z}+Q \frac{\partial^{2} u_{z}}{\partial z^{2}}+R \frac{\partial \in}{\partial y}=\frac{\partial^{2}}{\partial t^{2}}\left(\rho_{12} u_{z}+\rho_{22} U_{z}\right)-b \frac{\partial}{\partial t}\left(u_{z}-U_{z}\right),
\end{aligned}
$$

where $\left(u_{x}, u_{y}, u_{z}\right)$ and $\left(U_{x}, U_{y}, U_{z}\right)$ are displacements of the solid and liquid media, respectively, while $e$ and $\in$ are dilatations of the solid and liquid respectively; $A, N, Q, R, F, L, M$ and $C$ are all poroelastic constants and $\rho_{11}, \rho_{12}, \rho_{22}$ are the mass coefficients following Biot [11]. The stresses $\sigma_{i j}$ and the liquid pressure $s$ of the transversely isotropic poroelastic solid given by Biot [12] are

$$
\begin{aligned}
& \sigma_{x x}=P e_{x x}+A e_{y y}+F e_{z z}+M \in, \\
& \sigma_{y y}=A e_{x x}+P e_{y y}+F e_{z z}+M \in, \\
& \sigma_{z z}=F e_{x x}+F e_{y y}+C e_{z z}+Q \in, \\
& \sigma_{x y}=N e_{x y}, \\
& \sigma_{y z}=L e_{y z}, \\
& \sigma_{z x}=L e_{z x}, \\
& s=M e_{x x}+M e_{y y}+Q e_{z z}+R \in,
\end{aligned}
$$

The physical meaning of other parameters $A, N, Q, R, F, L, M$ and $C$ are given by Biot [3] as

$$
\begin{gathered}
P=\frac{E\left(1-\frac{E}{E^{\prime}} v^{\prime 2}\right)}{(1+v)\left(1-v-\frac{2 E}{E^{\prime}} v^{\prime 2}\right)}, \quad F=\frac{E v^{\prime}}{\left(1-v-\frac{2 E}{E^{\prime}} v^{\prime 2}\right)}, C=\frac{E^{\prime}(1-v)}{\left(1-v-\frac{2 E}{E^{\prime}} v^{\prime 2}\right)}, \\
L=G^{\prime}, \quad N=\frac{E}{2(1+v)} \text { and } M=\frac{R}{f^{2}} .
\end{gathered}
$$

where $P$ is a poroelastic constant given by $P=A+2 N$, the two constants $A, N$ correspond to familiar Lame constants in purely elastic solid, which are positive 
in sign. The coefficient $N$ represents the shear modulus of the solid. The coefficient $R$ is a measure of the pressure required on the liquid to force a certain amount of the liquid into the aggregate while total volume remains constant. The coefficient $Q$ represents the coupling between the volume change of the solid to that of liquid. $E$ are $E^{\prime}$ Young's moduli in the plane of transverse isotropy and in a direction normal to it, respectively. $v$ and $v^{\prime}$ are Poisson's ratios characterizing the lateral strain response in the plane of transverse isotropy to a stress acting parallel or normal to it, respectively. $G^{\prime}$ is the shear modulus in planes normal to the plane of transverse isotropy.

For Love waves, the displacement is only along $y$ direction thus the non-zero displacement component of the solid and liquid media are $(0, v, 0)$ and $(0, V, 0)$ respectively. These displacements are functions of $x, z$ and time $t$. Then the equations of motion of transversely isotropic poroelastic solid by Biot [11] that is Equation (1) reduces to

$$
\begin{aligned}
& N \frac{\partial^{2} v}{\partial x^{2}}+L \frac{\partial^{2} v}{\partial z^{2}}=\frac{\partial^{2}}{\partial t^{2}}\left(\rho_{11} v+\rho_{12} V\right)+b \frac{\partial}{\partial t}(v-V), \\
& 0=\frac{\partial^{2}}{\partial t^{2}}\left(\rho_{12} v+\rho_{22} V\right)-b \frac{\partial}{\partial t}(v-V) .
\end{aligned}
$$

We assume the propagation mode shapes of solid and liquid $u_{y}$ and $U_{y}$ are

$$
v=\phi(z) \mathrm{e}^{i(k x+\omega t)}, \quad V=\varphi(z) \mathrm{e}^{i(k x+\omega t)},
$$

where $t$ is time, $\omega$ is circular frequency, $k$ is wave number and $i$ is the complex unity.

Substitution of Equation (4) into Equation (3) yields

$$
\begin{aligned}
& L \phi^{\prime \prime}-k^{2} N \phi=-\omega^{2}\left(K_{11} \phi+K_{12} \varphi\right), \\
& 0=-\omega^{2}\left(K_{12} \phi+K_{22} \varphi\right),
\end{aligned}
$$

where

$$
K_{11}=\rho_{11}-\frac{i b}{\omega}, K_{12}=\rho_{12}+\frac{i b}{\omega}, K_{22}=\rho_{22}-\frac{i b}{\omega} .
$$

From the second equation of (5), we get

$$
\varphi=-\frac{K_{12}}{K_{22}} \phi
$$

Substitution of Equation (7) into the first equation of (5) we obtain,

$$
\phi^{\prime \prime}+\gamma^{2} \phi=0 \text {, }
$$

where

$$
\gamma^{2}=\left(-\frac{k^{2} N}{L}+\frac{\omega^{2} N}{L V_{3}^{2}}\right) \text { and } V_{3}^{2}=\left(\frac{N K_{22}}{K_{11} M_{22}-K_{12}^{2}}\right) .
$$

In Equation (9), $V_{3}$ is shear wave velocity as in Biot [11].

On simplification, Equation (8) gives 


$$
\phi(z)=C_{1} \mathrm{e}^{i \gamma z}+C_{2} \mathrm{e}^{-i \gamma z},
$$

where $C_{1}$ and $C_{2}$ are constants.

From Equation (7), $\varphi(z)$ can be obtained as

$$
g(z)=-\frac{M_{12}}{M_{22}}\left(C_{1} \mathrm{e}^{i \gamma z}+C_{2} \mathrm{e}^{-i \gamma z}\right) .
$$

Substituting $f(z)$ from Equation (10) into the first equation of (4), the displacement $u_{y}$ is

$$
v=\left(C_{1} \mathrm{e}^{i \gamma z}+C_{2} \mathrm{e}^{-i \gamma z}\right) \mathrm{e}^{i(k x+\omega t)} .
$$

Following Equations (2) and (11), the only non-zero stress can be obtained as

$$
\sigma_{y z}=\left(C_{1}\left(\frac{i \gamma L}{2}\right) \mathrm{e}^{i \gamma z}+C_{2}\left(\frac{i \gamma L}{2}\right) \mathrm{e}^{-i \gamma z}\right) \mathrm{e}^{i(k x+\omega t)} .
$$

From Equation (4), it can be shown that the normal strains of solid and liquid are zero, hence the dilatations of solid and liquid media vanish. Since the dilatations of solid and liquid are zero, the liquid pressure $s$ developed in the solid-liquid aggregate following Equation (2) is zero. Thus, no distinction is made between a pervious and an impervious surface of the solid in case of Love waves.

In the absence of body forces, the equations of motion [1] for viscous compressible liquid are

$$
\rho_{l}\left(\frac{\partial \boldsymbol{V}}{\partial t}\right)=-\nabla p+\frac{\eta_{l}}{3} \nabla(\nabla \cdot \boldsymbol{V})+\eta_{l} \nabla^{2} \boldsymbol{V},
$$

where $\boldsymbol{V}\left(u_{l}, v_{l}, w_{l}\right)$ is the velocity vector, $\rho_{l}$ is density of liquid, $\eta_{l}$ is coefficient of viscosity, $p$ is over pressure.

For Love waves, $\boldsymbol{V}=\left(0, v_{l}, 0\right)$ and $\nabla \cdot \boldsymbol{V}=\mathbf{0}$. Hence, Equation (13) reduces to

$$
\left(\frac{\partial v_{l}}{\partial t}\right)=\alpha_{l} \nabla^{2} v_{l}
$$

where $\alpha_{l}=\eta_{l} / \rho_{l}$ is the kinematic viscosity.

Solution of Equation (14) can be written as

$$
v_{l}=D \mathrm{e}^{\beta z} \mathrm{e}^{i(k x+\omega t)}
$$

where $\beta^{2}=k^{2}-\frac{i \omega}{\alpha_{l}}$ and $D$ is a constant.

Following (15), stresses in compressible viscous liquid layer can be shown as

$$
\tau_{y z}=D \eta_{l} \beta \mathrm{e}^{\beta z} \mathrm{e}^{i(k x+\omega t)} .
$$

\section{Frequency Equation}

For contact between the poroelastic layer and the viscous liquids, we assume that the stresses and displacement components are continuous at the interfaces $z=0$ and $z=h$.

Thus, the boundary conditions are given by 


$$
\begin{aligned}
& \text { at } z=0 ; \tau_{y z}^{(1)}=\sigma_{y z}, v_{l}^{(1)}=i \omega v \\
& \text { at } z=h ; \quad \sigma_{y z}=\tau_{y z}^{(2)}, i \omega v=v_{l}^{(2)} .
\end{aligned}
$$

Equation (17) results in a system of four homogeneous algebraic equations in four constants.

For a nontrivial solution, the determinant of the coefficients must vanish. By eliminating these constants the frequency equation of wave propagation in an isotropic poroelastic layer bounded by viscous liquid layers is

$$
\left|\begin{array}{cccc}
\eta_{f}^{(1)} \beta^{(1)} & \frac{i \gamma L}{2} & -\frac{i \gamma L}{2} & 0 \\
1 & i \omega & i \omega & 0 \\
0 & \frac{i \gamma L}{2} \mathrm{e}^{i \gamma h} & -\frac{i \gamma L}{2} \mathrm{e}^{-i \gamma h} & -\eta_{f}^{(2)} \beta^{(2)} \mathrm{e}^{-\beta^{2} h} \\
0 & i \omega \mathrm{e}^{i \gamma h} & i \omega \mathrm{e}^{-i \gamma h} & \mathrm{e}^{-\beta^{(2)} h}
\end{array}\right|=0 .
$$

On simplification Equation (18) reduces to

$$
\left[\gamma^{2} L^{2}+4 \omega^{2} \eta_{f}^{(1)} \beta^{(1)} \eta_{f}^{(2)} \beta^{(2)}\right] \tan (\gamma h)=2 i \omega \gamma L\left[\eta_{f}^{(1)} \beta^{(1)}+\eta_{f}^{(2)} \beta^{(2)}\right] .
$$

Equation (19) represents frequency equation of Love waves in a transversely isotropic poroelastic layer bounded between two viscous liquids.

If the upper liquid is inviscid i.e. $\eta_{l}^{(1)}=0$, then the above frequency Equation (19) reduces to

$$
\gamma L \tan (\gamma h)-2 i \omega \eta_{f}^{(2)} \beta^{(2)}=0 .
$$

Equation (20) represents frequency equation of Love waves in a transversely isotropic poroelastic layer in contact with a viscous liquid.

If both upper and lower liquids are inviscid then the frequency Equation (19) reduces to

$$
\sin (\gamma h)=0 .
$$

On simplification of Equation (21), we obtain the frequency $\omega$ as

$$
\omega=V_{3} \sqrt{\frac{l^{2} \pi^{2}}{h^{2}} \frac{L}{N}+k^{2}}, \text { where } l=1,2,3, \cdots
$$

Here $\omega$ represents frequency of Love waves in a transversely isotropic poroelastic layer when it is free from two viscous liquids.

\section{Numerical Work}

Frequency Equations (19)-(21) are investigated numerically by considering two distinct poroelastic materials with parameters $N=0.234, L=0.8$. The physical parameters of two viscous liquids are taken as $\rho_{1}=0.1, \eta_{1}=0.5$ and $\rho_{2}=1, \eta_{2}=2.5$. Poroelastic medium is dissipative in nature and thus the wave number $k$ is complex. The waves generated obey diffusion type process and therefore get attenuated. Let $k=k_{r}+i k_{p}$ where $k_{r}$ is real and $k_{i}$ is the imaginary part of the wave number $k$. The real and imaginary part of the wave number corresponds to propagation and attenuation of waves. Hence, the phase velocity 
$C_{p}$ and attenuation coefficient $\delta$ are, respectively

$$
C_{p}=\frac{\omega}{k_{r}} \text { and } \delta=\frac{k_{i}}{k_{r}} .
$$

The effect of presence of viscous liquids on phase velocity against wave number $\left(k_{r} h\right)$ in transversely isotropic poroelastic isotropic layer is depicted in Figure 2. Phase velocity is less when the solid layer is bounded between viscous liquids than it is free from the liquids. Thus, presence of viscous liquids reduces the phase velocity of Love waves in transversely isotropic poroelastic layer. Also, phase velocity decreases as wave number increases. A sudden decrease observed in phase velocity when $k_{r} h$ increases from 0.2 to 0.4 then onwards it decreases gradually. From Figure 3, a similar observation is found in case of poroelastic layer. In Figure 4, phase velocity against wavenumber is depicted for both transversely isotropic poroelastic layer and poroelastic layer when each is bounded between viscous liquids. It is observed that phase velocity for transversely isotropic

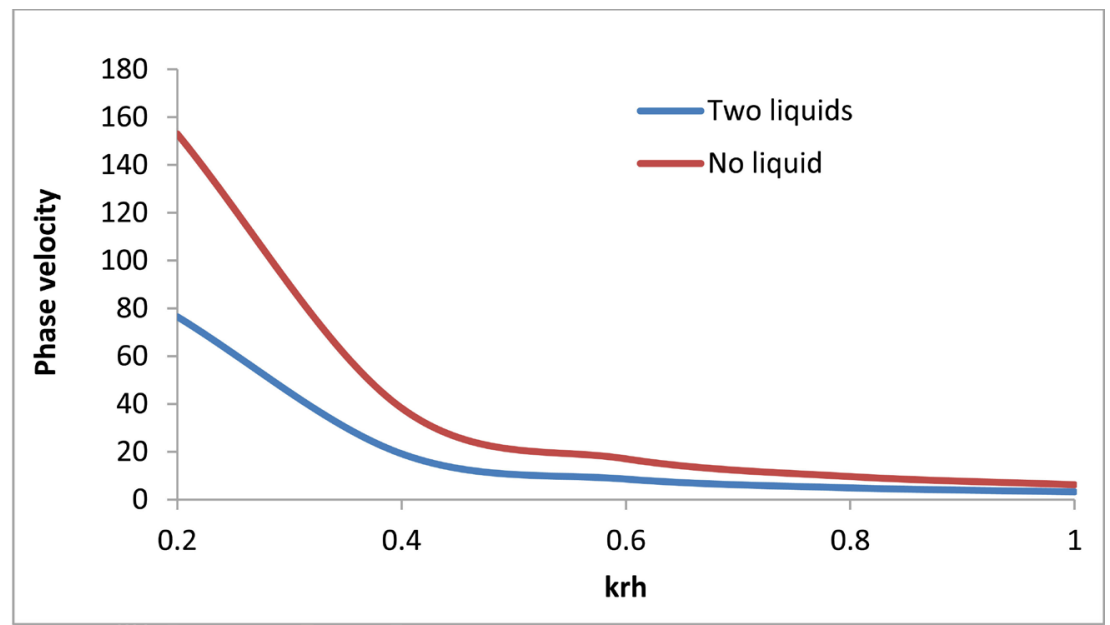

Figure 2. Phase velocity as a function of non-dimensional wave number-transversely isotropic poroelastic solid layer.

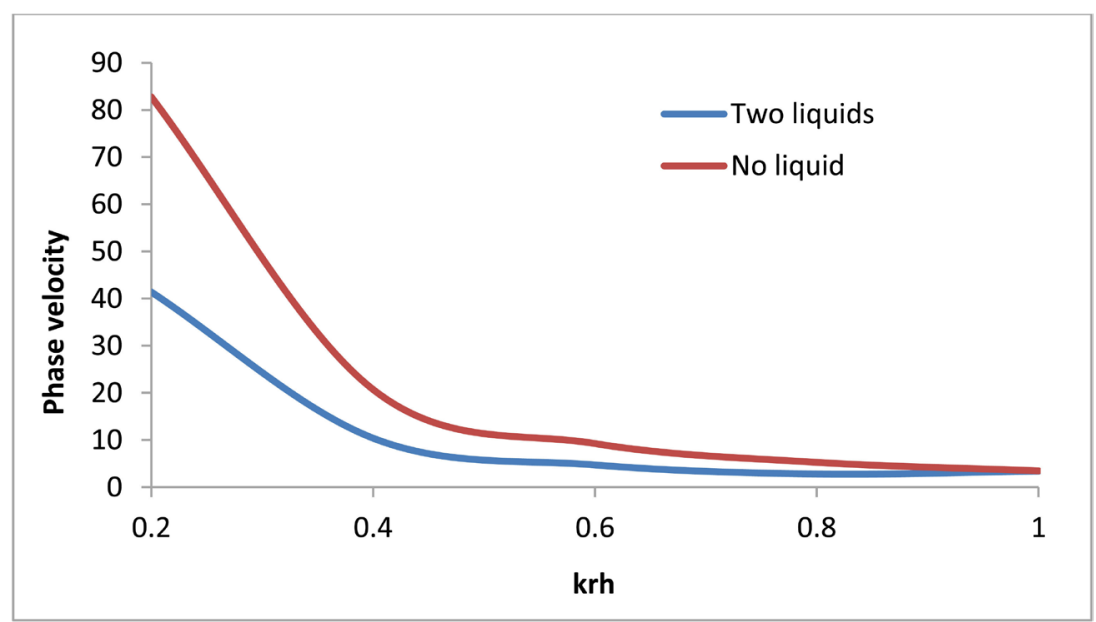

Figure 3. Phase velocity as a function of non-dimensional wave number-poroelastic solid layer. 
poroelastic layer is more compared to poroelastic layer. Phase velocity decreases as wave number increases and a sudden decrease observed in phase velocity when $k_{r} h$ increases from 0.2 to 0.4 then onwards it decreases gradually. Phase velocity at the interface of solid layer and lower viscous liquid is plotted in Figure 5 for both transversely isotropic poroelastic layer and poroelastic layer. Phase velocity for transversely isotropic poroelastic layer is very much higher than that of poroelastic layer. A sudden decrease observed in phase velocity when $k_{r} h$ increases from 0.2 to 0.4 and then from 0.6 to 1 and phase velocity increases when $k_{r} h$ increases from 0.4 to 0.6 . Phase velocity against wave number in the absence of liquids is presented in Figure 6. A sudden decrease is observed in phase velocity when $k_{r} h$ increases from 0.2 to 0.4 then onwards it decreases gradually. Attenuation as a function of non-dimensional wave number is depicted in Figure 7 for transversely isotropic poroelastic layer bounded by viscous liquids for different values of phase velocity i.e. $m=3,5,10$ and 15 . It is ob served

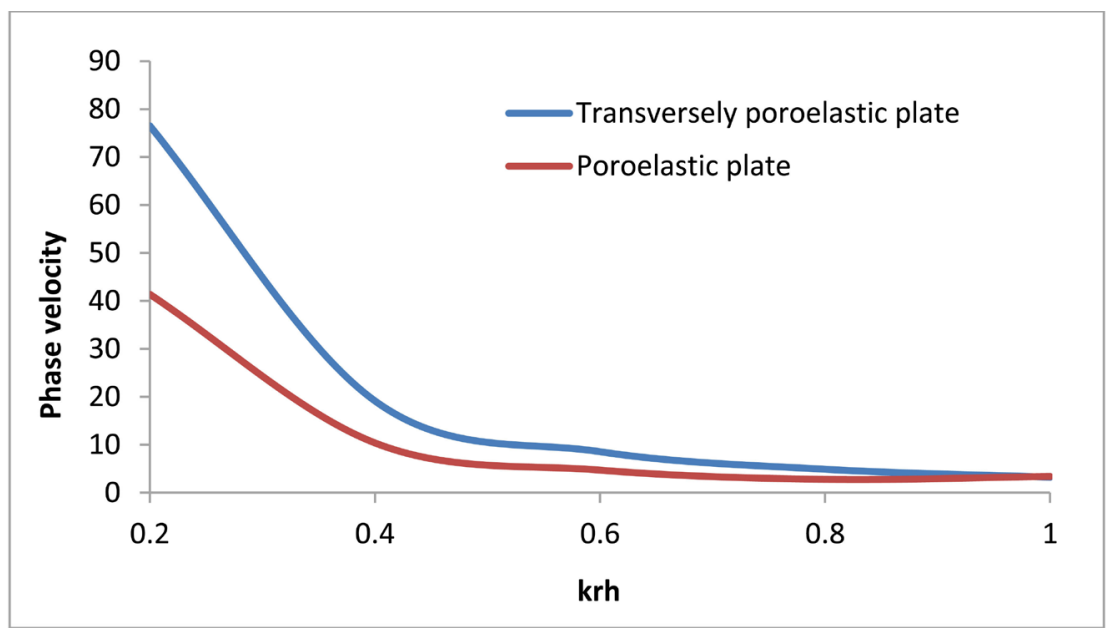

Figure 4. Phase velocity as a function of non-dimensional wave number-solid layer bounded between two viscous liquids.

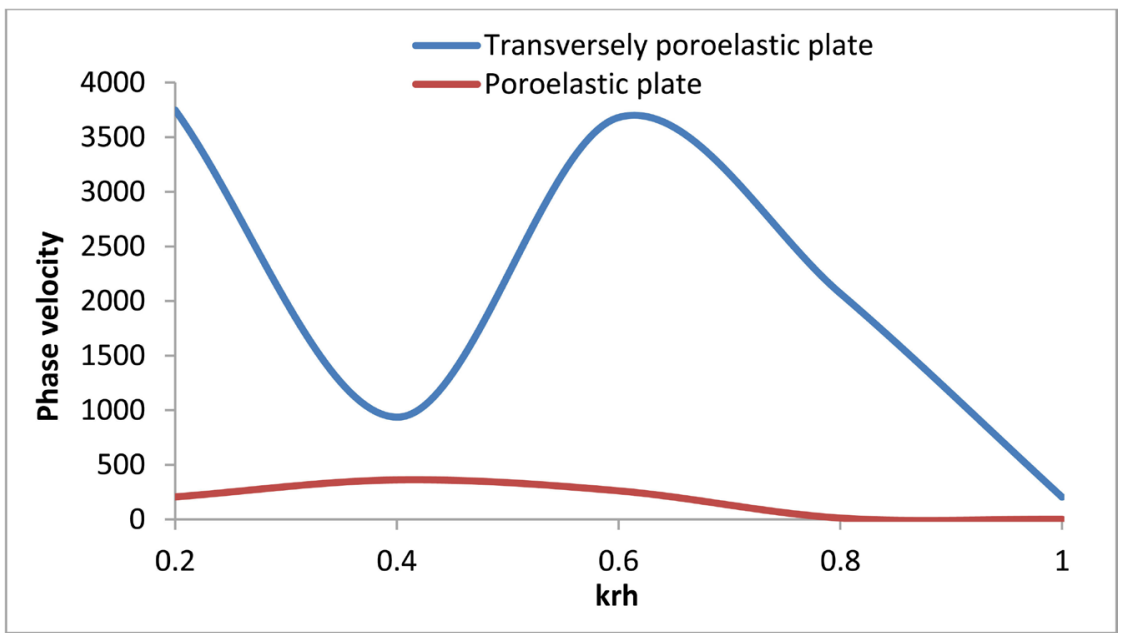

Figure 5. Phase velocity as a function of non-dimensional wave number-solid layer in contact with viscous liquid. 


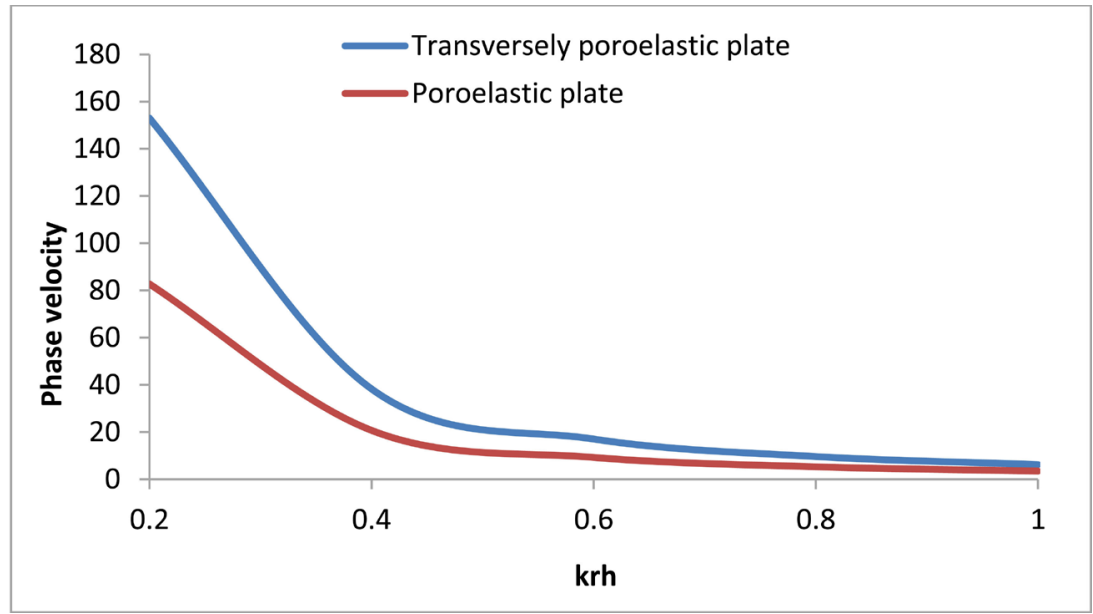

Figure 6. Phase velocity as a function of non-dimensional wave number-solid layer.

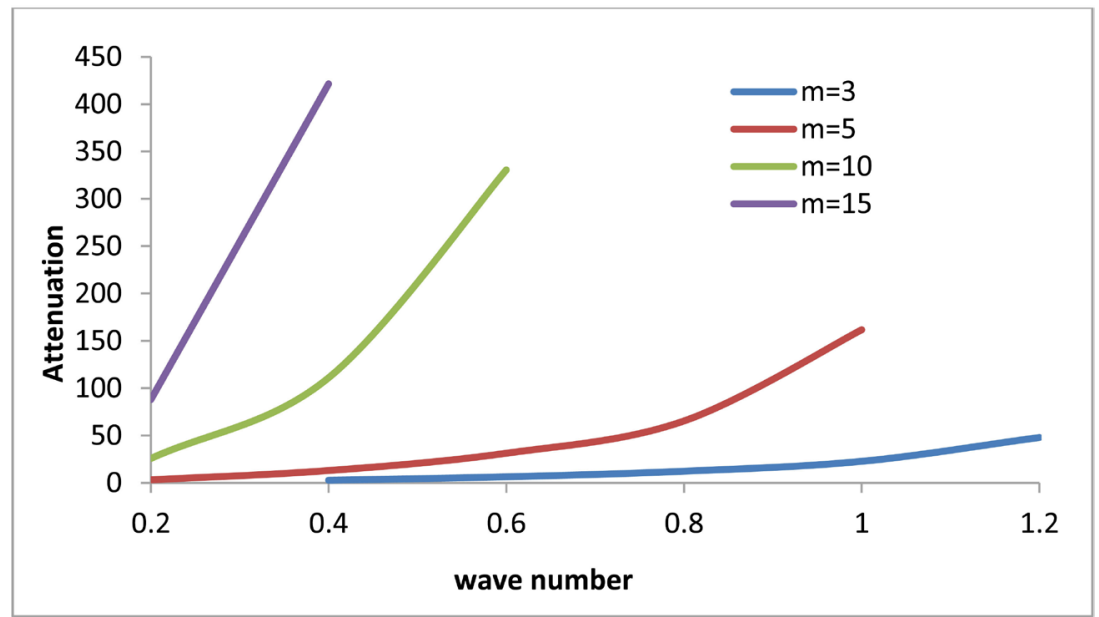

Figure 7. Attenuation as a function of non-dimensional wave number-transversely isotropic solid layer bounded between two viscous liquids.

that attenuation increases for higher values of phase velocity. A similar phenomenon is noticed in the case of poroelastic layer bounded by viscous liquids from Figure 8. But the attenuation for poroelastic layer is less when compared to transversely poroelastic layer.

\section{Conclusions}

A study of propagation of Love waves in an infinite poroelastic layer bounded by viscous liquids leads to the following conclusions:

1) Presence of upper and lower viscous liquids decreases phase velocity of Love waves for both transversely isotropic poroelastic layer and poroelastic layer.

2) Phase velocity of Love waves is more in transversely isotropic poroelastic layer than in poroelastic layer when they are bounded between viscous liquids as well as when they are free from viscous liquids. In both cases phase velocity decreases gradually.

3) Phase velocity of Love waves in transversely isotropic poroelastic layer is 


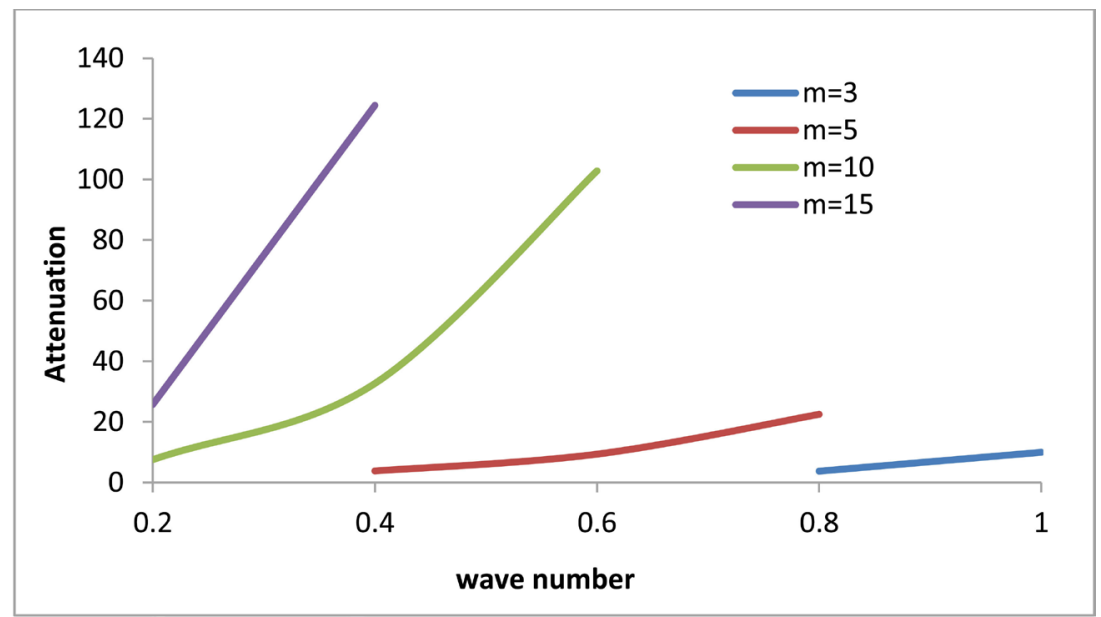

Figure 8. Attenuation as a function of non-dimensional wave number-poroelastic solid layer bounded between two viscous liquids.

very much higher than that of poroelastic layer when they are in contact with lower viscous liquid.

4) Attenuation in transversely isotropic poroelastic layer when it is bounded by viscous liquids is more when compared to the case of poroelastic layer bounded by viscous liquids. In both the cases, attenuation increases as phase velocity and wave number increase.

\section{Conflicts of Interest}

The authors declare no conflicts of interest regarding the publication of this paper.

\section{References}

[1] Deresiewicz, H. (1960) The Effect of Boundaries on Wave Propagation in a Liquid-Filled Porous Solid-I. Bulletin of the Seismological Society of America, 50, 599-607.

[2] Deresiewicz, H. and Rice, J.T. (1962) The Effect of Boundaries on Wave Propagation in a Liquid-Filled Porous Solid-II. Bulletin of the Seismological Society of America, 52, 595-626.

[3] Banghar, A.R. (1978) On Propagation and Attenuation of Love Waves. Proceedings of the Indian Academy of Sciences, 88, 133-146. https://doi.org/10.1007/BF02871610

[4] Nageswara Nath, C., Manoj Kumar, J. and Tajuddin, M. (2011) On the Parametric model of Loose Bonding between Two Poroelastic Half Spaces. Journal of Vibration and Control, 18, 1261-1274.

[5] Kiełczynski, P., Szalewski, M.A. and Alcerzak, B. (2012) Effect of a Viscous Liquid Loading on Love Wave Propagation. International Journal of Solids and Structures, 49, 2314-2319. https://doi.org/10.1016/j.ijsolstr.2012.04.030

[6] Anjana, P.G., Samal, S.K. and Mahanti, N.C. (2010) love Waves in a Fluid-Saturated Porous Layer under a Rigid Boundary and Lying over an Elastic Half-Space under Gravity. Applied Mathematical Modelling, 34, 1873-1883.

https://doi.org/10.1016/j.apm.2009.10.004 
[7] Wang, Y.S. and Zhang, Z.M. (1998) Propagation of Love Waves in a Transversely Isotropic Fluid Saturated Porous Layered Half-Space. Journal of the Acoustical Society of America, 103, 695-701. https://doi.org/10.1121/1.421196

[8] Nageswaranath, C., Manoj kumar, J. and Ahmed Shah, S. (2017) Effect of Viscosity on Waves Propagating in a Liquid Loaded on Poroelastic Layered Half-Space, Advanced Math. Models \& Applications, 2, 144-154.

[9] Nageswara Nath C., Manoj Kumar, J. and Ahmed Shah, S. (2015) On Propagation of Love Waves in an Infinite Transversely Isotropic Poroelastic Layer. Journal of Physics: Conference Series, 662, Article ID: 012004. https://doi.org/10.1088/1742-6596/662/1/012004

[10] Kundu, S., Manna, S. and Gupta, S. (2014) Love Wave Dispersion in Pre-Stressed Homogeneous Medium over a Porous Half-Space with Irregular Boundary Surfaces. International Journal of Solids and Structures, 51, 3689-3697.

[11] Biot, M.A. (1956) The Theory of Propagation of Elastic Waves in Fluid-Saturated Porous Solid. Journal of the Acoustical Society of America, 28, 168-178. https://doi.org/10.1121/1.1908239

[12] Biot, M.A. and Willis, D.G. (1957) The Elastic Co-Efficients of the Theory of Consolidation. Journal of Applied Mechanics, 24, 594-601. 